\title{
Leistungsausweis chirurgisch tätiger Ärzte in keiner Weise gewürdigt
}

\author{
Die FMCH (Foederatio Medicorum Chirurgicorum Helvetica), der Verband \\ chirurgisch tätiger Ärzte in der Schweiz, wehrt sich mit aller Vehemenz gegen \\ das unvollständige Positionspapier zur Qualitätssicherung von santésuisse.
}

Das im Januar vom Dachverband der Krankenversicherungen veröffentlichte Papier würdigt in keiner Weise die entscheidenden Beiträge der chirurgisch tätigen Ärztinnen und Ärzte in der Schweiz.

Für diese war und ist Qualitätssicherung im Rahmen von Weiter- und Fortbildung das Thema schlechthin, und dies seit mehr als 100 Jahren. Hinzu kommen systematische Datenerfassungen und -auswertungen (z.B. AQC, ASF, SwissVasc usw.), Qualitätssicherungserhebungen (z.B. Outcome), Critical Incident Reporting Systems (CIRS) und vieles andere mehr. Wenn nun santésuisse ein Positionspapier zum Thema Qualitätssicherung veröffentlicht und dabei mit keinem Wort die entscheidenden Beiträge der Schweizer Chirurgen erwähnt, zeugt dies von einer befremdenden Ignoranz den zentralen Anliegen der chirurgisch tätigen Ärzteschaft gegenüber. Die allermeisten von santésuisse geforderten Massnahmen werden nämlich von den invasiv tätigen Ärzten bereits seit vielen Jahren erfüllt.

\section{Brief der FMCH \\ an die Direktion von santésuisse, \\ lic. rer. pol. Marc-André Giger}

Sehr geehrter Herr Giger

Die FMCH, der Dachverband der chirurgisch tätigen Ärzte der Schweiz, hat das am 13. Januar 2006 publizierte Positionspapier der santésuisse bezüglich Qualitätssicherung zur Kenntnis genommen. Als Vertreter der Leistungserbringer, welche im Segment mit den höchsten Risiken (invasiv Tätige) arbeiten, fühlen wir uns verpflichtet, dazu Stellung zu nehmen.

Die gesetzlichen Grundlagen sind uns bekannt, und es ist uns ein grosses Anliegen und in unserem ureigenen Interesse, dass gesetzliche Rahmenbedingungen für die Sicherung der Qualität formuliert sind und umgesetzt werden.

Wir möchten darauf hinweisen, dass die invasiv tätige und operativ tätige Ärzteschaft auf dem
Gebiet der Qualitätssicherung immer sehr aktiv war und eine eigentliche Vorreiterrolle innehat. Ohne die selbstkritische Überprüfung unseres Tuns wäre der in den letzten 100 Jahren erzielte Fortschritt in der operativen Medizin nicht möglich gewesen und der heutige ausgezeichnete Stand der Gesundheitsversorgung unserer Bevölkerung nicht erreicht worden.

Es erstaunt deshalb, dass Sie in Ihrem Abschnitt über den Stand der Umsetzung der Qualitätssicherung mit keinem Wort diesen entscheidenden Beitrag der chirurgisch tätigen Ärztinnen und Ärzte erwähnen. Sie beschränken sich auf die Aufzählung von einigen Projekten, welche zum Teil am Anfang stehen und, ausser der gebildeten Kommission, noch wenig konkrete Aktivitäten aufzuweisen haben. Im Gegensatz dazu stehen die unzähligen Aktivitäten der invasiv tätigen Ärzteschaft.

Bei der Einführung einer vergleichbaren und messbaren Qualitätssicherung geht es letztlich auch um die Definition der Qualitätssicherung und um die Definition des Anforderungsprofils an diese Qualitätssicherung.

Aus unserer Sicht ist der eigentliche Garant für die Aufrechterhaltung einer sehr guten Qualität in den operativen Fächern die Aufrechterhaltung einer qualitativ hochstehenden Weiter- und Fortbildung. Eine hohe Weiterbildungsqualität wird nur da erreicht, wo in einem transparenten und offenen Vertrauensverhältnis die jungen Kollegen heranwachsen. Deshalb ist einer der wesentlichen Bestandteile der Qualitätssicherung die Weiterbildungsstätte mit ihren täglichen Rapporten, Chefvisiten und Weiterbildungen sowie der direkten 1:1-Unterrichtung der jungen Kollegen. Diese Praxis ist in den in der Schweiz anerkannten Weiterbildungsstätten für die operative Medizin eine gepflegte und sehr gut funktionierende Grundlage. Weiter bestehen im Rahmen der wissenschaftlichen Fortbildungen, organisiert durch die Fachgesellschaften, 
nationale und internationale Fortbildungsveranstaltungen, welche die neusten Erkenntnisse zeitnah und fachkompetent vermitteln.

Solcher Informationsaustausch kann nur auf ausgewerteten Patientendaten beruhen. Eine ausgezeichnete Datenlage ist in der operativen Medizin unabdingbar, um im Feld der Weiterentwicklung und der Qualitätssicherung Schlüsse ziehen zu können. Mehrere chirurgische Fachgesellschaften haben zudem Qualitätssicherungsprogramme, in welchen entweder das gesamte Patientengut systematisch erfasst wird oder gezielt einzelne Diagnosegruppen systematisch nachuntersucht und nachkontrolliert werden. Auf diese Weise können einerseits im Bereich von Einzeldiagnosen wesentliche Fortschritte erzielt werden, andererseits dienen systematische Datenerfassungen, wie sie z. B. die Schweizerische Gesellschaft für Chirurgie mit der AQC pflegt (AQC = Arbeitsgemeinschaft für Qualitätssicherung in der Chirurgie), dem Vergleich (Benchmark) zwischen den einzelnen Datenlieferanten und Spitälern. Weiter haben unseres Wissens die Spitäler und Leistungserbringer jederzeit bei kantonal oder national initiierten Qualitätssicherungserhebungen (z.B. Outcome) mitgemacht und so wesentliche Beiträge für die Förderung dieser Qualitätssicherungsbestrebungen geleistet. Freiwillig und in der Regel ohne Beantragung zusätzlicher Ressourcen haben viele Spitäler, ebenfalls motiviert durch die operativ tätige Ärzteschaft, sogenannte Critical Incident Reporting Systems (CIRS) etabliert und auf diese Weise auch zur Verbesserung der Fehlerkultur in den Spitälern beigetragen.

Wenn Sie diesen Leistungsausweis der invasiv Tätigen der Position der santésuisse gegenüber- stellen, wird klar, dass fast alles, was die santésuisse fordert, bereits durch die Ärzteschaft etabliert wurde. Es ist mehr als bedauerlich und demotivierend, dass diese Tatsachen in Ihrem Positionspapier keine Beachtung finden.

Uns besorgt bei der Position der santésuisse nicht die eigentliche Absicht, die Qualität zu messen und $\mathrm{zu}$ vergleichen, sondern die dahinter verborgenen ökonomischen Fallen, Entsolidarisierung, Risikoselektion, Zweiklassenmedizin, Priorisierung der Ökonomie vor die eigentliche Medizin und die sogenannten Märkte (Wettbewerb), um nur einige zu nennen.

Wir möchten mit unserer Stellungnahme verhindern, dass eine reine Verpolitisierung des Qualitätsanliegens in der Medizin geschieht und als Folge Steuerungsmechanismen in Gang gesetzt werden, die dem medizinischen Fortschritt entgegenwirken und ethisches Verhalten erschweren. Auf der anderen Seite unterstützen wir, unserer Tradition entsprechend, die transparente Offenlegung unserer Leistungen mit dem Ziel, die Qualität der Versorgung unserer Patienten weiter zu verbessern. Wir sehen uns klar als Ansprechpartner der chirurgisch tätigen Ärzteschaft in der gesundheitspolitischen Diskussion und bei der Entwicklung der Qualitätssicherung und suchen diesbezüglich auch den aktiven Dialog.

Mit freundlichen Grüssen

Prof. Dr. med. Urban Laffer, Präsident FMCH Prof. Dr. med. Othmar Schöb, Ressortleiter Qualitätssicherung FMCH 